\title{
COMUNICAÇÃOCIENTÍFICA
}

\section{DISTRIBUIÇÃO DO SISTEMA RADICULAR DA BANANEIRA 'PRATA-ANÃ' EM DUAS FREQÜÊNCIAS DE FERTIRRIGAÇÃO COM URÉIA ${ }^{1}$}

\author{
ANA LÚCIA BORGES², LUCIANO DA SILVA SOUZA², CÉSAR AUGUSTO BASTOS PEIXOTO², \\ JORGE LUIS COPQUER DOS SANTOS JÚNIOR ${ }^{4}$
}

RESUMO - O conhecimento da distribuição das raízes da bananeira subsidia informações para orientar a melhor localização de água e fertilizantes, resultando em incremento da produtividade do pomar. Em Latossolo Amarelo distrófico argissólico, em sistema de fileiras duplas ( 4 × 2 × 2 m), avaliou-se a distribuição do sistema radicular da bananeira 'Prata-Anã', antes da colheita do $2^{\circ}$ ciclo, em duas freqüências de fertirrigação com uréia ( $400 \mathrm{~kg}$ de N/ha/ano), a cada 3 dias (F1) e a cada 15 dias (F2). As raízes foram coletadas em diferentes profundidades e distâncias, utilizando tubo metálico, sendo separadas do solo e quantificadas pelo programa GS Root. A maior freqüência de aplicação de $\mathrm{N}$ e de água (3 dias) favoreceu a densidade de raízes, em comparação com a menor freqüência (15 dias). A maior concentração de raízes ocorreu nas camadas superficiais, até $0,30 \mathrm{~m}$, e entre a planta e o microaspersor. Predominaram raízes de diâmetro entre $0,2 \mathrm{e}>1,5 \mathrm{~mm}$, tanto nas camadas superficiais ( 0 a $0,20 \mathrm{~m}$ de profundidade) quanto entre a planta e o microaspersor. Termos para indexação: Musa spp., nitrogênio, sentido do microaspersor, profundidade.

\section{DISTRIBUTION OF THE 'PRATA ANÃ' ROOT SYSTEM IN TWO UREA FERTIRRIGATION FREQUENCIES}

\begin{abstract}
The knowledge of the distribution of roots in bananas generates information that guide the location of water and fertilizers, resulting an increase in crop yield in banana plantations. The 'Prata Anã' banana root distribution system, in loam clay sandy Yellow Latosol, before harvest of the second cycle, was evaluated in two urea fertirrigation frequencies ( $400 \mathrm{~kg}$ of N/ha/year) every 3 days (F1) and 15 days (F2). Root samples were collected in different distances and depths with a metallic tube, separated from the soil and quantified using the GSRoot software. Higher urea fertirrigation frequency ( 3 days) favored root density, in relation to the 15 days frequency. The amount of roots in the superficial layers (up to $0.30 \mathrm{~m}$ ) was greater, decreasing with the depth, as well as for the plant in the direction of the micro sprinkler, and less in the opposite direction. Roots of diameter between 0.2 to $>1.5 \mathrm{~mm}$ prevailed in the superficial layers and beside the plant in the direction of the micro sprinkler.
\end{abstract}

Index terms: Musa spp., nitrogen, micro sprinkler direction, depth.

Em áreas irrigadas, é fundamental o conhecimento das inter-relações entre água-solo-planta-clima para o manejo adequado da irrigação e da fertirrigação. A extensão do sistema radicular é resultado do potencial genético da planta, além de fatores ambientais. Avilan et al. (1982), avaliando a distribuição do sistema radicular da bananeira sob diferentes sistemas de preparo e manejo do solo (subsolagem, aração, camalhão), verificaram que $100 \%$ das raízes se concentraram até $0,50 \mathrm{~m}$ de profundidade e $90 \%$ até $0,30 \mathrm{~m}$ de distância do pseudocaule, em solo franco-argiloso a franco-siltoso; já em solos mais argilosos, as raízes concentraram-se a $0,80 \mathrm{~m}$ de profundidade e aproximadamente $50 \%$ a $0,60 \mathrm{~m}$ de distância do pseudocaule. Em solo areia franca irrigado por microaspersão, Garcia (2000) verificou que $60 \%$ do sistema radicular da bananeira 'Prata-Anã' se concentraram nos primeiros $0,30 \mathrm{~m}$ de profundidade e $70 \%$ no sentido do microaspersor; $72 \%$ da massa seca das raízes foram classificadas como grossas (percepção visual) e 78\% localizadas de 0,05 a $0,35 \mathrm{~m}$ do pseudocaule. Na irrigação convencional, $95 \%$ concentraram-se na camada de 0 a $0,30 \mathrm{~m}$ de profundidade e com
91\% de raízes grossas. Para a variedade Pacovan, com 26 meses de idade, irrigada por microaspersão, Montenegro et al. (2004) constataram, em um Cambissolo de textura franco-argilosa, maior concentração de raízes (45\%) a $0,40 \mathrm{~m}$ de distância do pseudocaule e $94 \%$ a $0,40 \mathrm{~m}$ de profundidade. $\mathrm{O}$ trabalho objetivou determinar a distribuição do sistema radicular da bananeira 'Prata-Anã' em duas freqüências (3 e 15 dias) de fertirrigação com uréia.

Desenvolveu-se o trabalho na área experimental da Embrapa Mandioca e Fruticultura Tropical, no Município de Cruz das Almas, Região do Recôncavo Baiano, a 12 40'19” de latitude sul, 3906'22" de longitude oeste Gre altitude de $220 \mathrm{~m}$. O clima da região é subúmido, com temperatura média anual de $24^{\circ} \mathrm{C}$, umidade relativa do ar média anual de $80 \%$ e precipitação média anual de $1.200 \mathrm{~mm}$ (Souza \& Souza, 2001). O solo da área experimental é um Latossolo Amarelo distrófico argissólico (306 g de argila, $126 \mathrm{~g}$ de silte e $568 \mathrm{~g}$ de areia/ $\mathrm{kg}$, na camada de $0-0,20 \mathrm{~m}$, textura francoargiloarenosa) e com os seguintes atributos químicos nessa profundidade: $\mathrm{pH}$ em água $=5,5 ; \mathrm{P}=8 \mathrm{mg} / \mathrm{dm}^{3} ; \mathrm{K}=138 \mathrm{mg} / \mathrm{dm}^{3}$; $\mathrm{Ca}=3,1 \mathrm{cmol} / \mathrm{dm}^{3} ; \mathrm{Mg}=1,0 \mathrm{cmol} / \mathrm{dm}^{3}, \mathrm{eV}=65 \%$. Estudou-se a

1(Trabalho 020-07). Recebido em: 15-01-2007. Aceito para publicação em : 07-01-2008.

${ }^{2}$ Eng (a). Agr (a), Pesquisador (a) da Embrapa Mandioca e Fruticultura Tropical. Caixa Postal 007, CEP 44380-000 Cruz das Almas-BA. E-mails: analucia@cnpmf.embrapa.br, 1souza@cnpmf.embrapa.br.

${ }^{3}$ Aluno de graduação do Centro de Ciências Agrárias e Ambientais da UFRB, Cruz das Almas-BA. E-mail: cabpagr@yahoo.com.br.

${ }^{4}$ Mestrando em Ciências Agrárias da UFRB, Cruz das Almas-BA. E-mail: jorgecopquer@yahoo.com.br. 
bananeira 'Prata-Anã', cultivada em sistema de fileiras duplas, no espaçamento de $4 \times 2 \times 2$ m e fertirrigada a partir do primeiro mês com uréia, na dose fixa de $400 \mathrm{~kg}$ de N/ha e mesma lâmina de água, em duas freqüências de fertirrigação: a cada três dias (F1) e a cada quinze dias (F2), anualmente. O potássio, na forma de $\mathrm{KCl}(150 \mathrm{~kg}$ de $\mathrm{K}_{2} \mathrm{O} / \mathrm{ha} / \mathrm{ano}$ ), foi aplicado semanalmente, também via água de irrigação. A aplicação de $\mathrm{N}$ e K foi feita por microaspersão, sendo um emissor para cada quatro plantas, com vazão de $43 \mathrm{~L} / \mathrm{h}$. A lâmina de água foi calculada com base na transpiração potencial estimada por Penman modificado, numa estação meteorológica automática. As amostras de raízes foram coletadas em duas plantas adultas, em cada freqüência, utilizando tubo metálico com $0,085 \mathrm{~m}$ de diâmetro e $1,20 \mathrm{~m}$ de comprimento, em nove distâncias do microaspersor $(0 ; 0,375 ; 0,750 ; 1,125 ; 1,500 ; 1,850 ; 2,225 ; 2,600 \mathrm{e}$ $2,975 \mathrm{~m}$ ), com a planta localizada a 1,675 m do microaspersor, até a profundidade de $0,80 \mathrm{~m}$, em intervalos de $0,10 \mathrm{~m}$, totalizando-se 288 amostras. As raízes foram separadas do solo, digitalizadas e quantificadas pelo programa GSRoot (Guddanti \& Chambers, 1993). O programa permitiu determinar o comprimento total de raízes de cada amostra, separando nas seguintes classes de diâmetro (mm): $<0,1 ; 0,1-0,2 ; 0,2-0,3 ; 0,3-0,5 ; 0,5-0,8 ; 0,8-1,5 \mathrm{e}>1,5$, sendo os dados finais expressos em densidade de raízes ( $\mathrm{cm}$ de raiz/ $\mathrm{dm}^{3}$ de solo) para as sete classes de diâmetro, computandose também a densidade total de raízes envolvendo todos os diâmetros. Os dados obtidos foram avaliados, como delineamento experimental inteiramente casualizado, em esquema fatorial $2 \times 9 x$ 8 (freqüência $x$ distância $x$ profundidade). Inicialmente, foi avaliada a normalidade dos dados para as oito variáveis, utilizando o teste de Kolmogorov-Smirnov, observando-se que todas elas não seguiram a distribuição normal. Em função disso, para procedimento de análise estatística, os dados foram transformados em $\log _{\mathrm{e}}(\mathrm{x}+2)$ e comparados com relação à freqüência de fertirrigação e à profundidade e distância do microaspersor, e também para suas interações. Os dados médios foram comparados por meio do teste de Tukey, a $5 \%$ de probabilidade, para cada classe de diâmetro e para a densidade total de raízes. As análises estatísticas foram realizadas por meio do aplicativo SAS (SAS Institute, 1990). Ajustou-se também uma superfície de resposta com os valores da densidade total de raízes, utilizando o aplicativo Surfer versão 7.0, apresentando a distribuição espacial das raízes em relação à profundidade e a distância do microaspersor, separadamente para cada freqüência de fertirrigação.

A análise de variância dos dados mostrou significância estatística $(p<0,01)$ entre as duas freqüências de fertirrigação, com superioridade para a freqüência de 3 dias, em todas as classes de diâmetro de raízes avaliadas (Tabela 1). Credita-se isso à disponibilidade mais freqüente de $\mathrm{N}$ e de água para as plantas, na freqüência de 3 dias. A maior quantidade de nitrogênio aplicada por evento, na freqüência de 15 dias, proporcionou maior lixiviação desse nutriente, principalmente devido ao fato de o solo conter teor de areia acima de $500 \mathrm{~g} / \mathrm{kg}$.

Tanto para a freqüência de 3 dias quanto para a de 15 dias, houve decréscimo na densidade de raízes ao longo do perfil (Figuras 1A e 1B). A distribuição espacial da densidade total de raízes em relação à profundidade e a distância do microaspersor mostrou, nas duas freqüências, maior concentração de raízes até $0,30 \mathrm{~m}$ de profundidade e nas distâncias de $0,375 \mathrm{~m}$ e $1,500 \mathrm{~m}$ do microaspersor; a densidade de raízes nas distâncias a partir de 2,250 $\mathrm{m}$ foi bem menor, principalmente a partir de $0,40 \mathrm{~m}$ de profundidade (Figuras 1Ae 1B). A profundidade efetiva do sistema radicular, até onde se encontraram pelo menos $80 \%$ das raízes, foi de 0,50 e $0,60 \mathrm{~m}$, para as freqüências de 3 e 15 dias, respectivamente. Considerando a profundidade de $0,30 \mathrm{~m}$, como discutido por Garcia (2000), 60\% das raízes concentraram-se nessa profundidade, nas duas freqüências, valores semelhantes ao obtido por esse autor. Associa-se esse comportamento às condições mais favoráveis existentes nas camadas mais próximas à superfície do solo, onde a atividade biológica e a disponibilidade de água e nutrientes são maiores. Resultado diferente deste estudo foi constatado por Avilan et al. (1982), que verificaram $100 \%$ das raízes até $0,50 \mathrm{~m}$ em solos mais arenosos e sistema radicular mais profundo nos argilosos.

Quanto a distância do microaspersor, observou-se maior concentração de raízes entre a planta e o micraspersor $(71 \%$ em F1 e 65\% em F2), com uma distribuição mais uniforme da densidade total de raízes em todas as distâncias, na freqüência de 15 dias (Figura 1B). Entre a planta e o microaspersor, as quantidades de água e de $\mathrm{N}$ estão em maior quantidade e mais bem distribuídas, favorecendo o desenvolvimento do sistema radicular. Valores semelhantes foram observados por Garcia (2000), o que deve estar associado à maior disponibilidade de água. Observou-se predominância de raízes com diâmetro entre $0,2 \mathrm{a}>1,5 \mathrm{~mm}(76 \%)$, notadamente nas camadas superficiais, até $0,20 \mathrm{~m}$ (Tabela 2), e entre a planta e o microaspersor (Tabela 3). Nas camadas mais profundas, ocorreu redução das raízes mais grossas, passando a predominar os diâmetros de 0,2-0,5 mm, embora as raízes com diâmetro $>1,5 \mathrm{~mm}$ tenham sido encontradas em todas as profundidades avaliadas (Tabela 2). Do lado da planta oposto ao microaspersor, ocorreu redução relativamente uniforme em toda a faixa de diâmetro de $0,2 \mathrm{a}>1,5 \mathrm{~mm}$ (Tabela 3 ).

Assim, pode-se concluir com o trabalho: a) a maior freqüência de aplicação de $\mathrm{N}$ e de água (3 dias) favoreceu a densidade de raízes, em comparação com a menor freqüência (15 dias); b) a maior concentração de raízes ocorreu nas camadas superficiais, até $0,30 \mathrm{~m}$, e entre a planta e o microaspersor; c) predominaram raízes de diâmetro entre $0,2 \mathrm{a}>1,5 \mathrm{~mm}$, tanto nas camadas superficiais quanto entre a planta e o microaspersor.

TABELA 1- Densidade de raízes da bananeira 'Prata-Anã', por classes de diâmetro, em função da freqüência de fertirrigação com uréia. Média de oito profundidades e nove distâncias do microaspersor. Cruz das Almas-BA.

\begin{tabular}{|c|c|c|c|c|c|c|c|c|}
\hline \multirow{2}{*}{$\begin{array}{l}\text { Frequiência } \\
\text { (dias) }\end{array}$} & \multicolumn{8}{|c|}{ Diâmetro das raízes $(\mathrm{mm})$} \\
\hline & D1 & D2 & D3 & D4 & D5 & D6 & D7 & TOTAL \\
\hline \multicolumn{9}{|c|}{-- Densidade de raízes $\left(\mathrm{cm}\right.$ de raiz $/ \mathrm{dm}^{3}$ de solo) } \\
\hline 3 & $8,2 \mathrm{a}$ & $16,7 \mathrm{a}$ & $102,2 \mathrm{a}$ & 94,9 a & $78,0 \mathrm{a}$ & $70,0 \mathrm{a}$ & $90,3 \mathrm{a}$ & $460,3 \mathrm{a}$ \\
\hline 15 & $6,8 \mathrm{~b}$ & $12,8 \mathrm{~b}$ & $71,4 b$ & $46,3 \mathrm{~b}$ & $35,6 \mathrm{~b}$ & $35,1 \mathrm{~b}$ & $29,7 \mathrm{~b}$ & $237,8 \mathrm{~b}$ \\
\hline $\mathrm{CV}(\%)$ & 29.4 & 26,4 & 19,1 & 18,9 & 18,8 & 20,2 & 24,4 & 13,8 \\
\hline
\end{tabular}

${ }^{1}$ Classes de diâmetro $(\mathrm{mm}): \mathbf{D} 1=<0,1 ; \mathbf{D} 2=0,1-0,2 ; \mathbf{D 3}=0,2-0,3 ; \mathbf{D 4}=0,3-0,5$; D5 $=0,5-0,8 ; \mathbf{D} 6=0,8-1,5$ e $\mathbf{D} 7=>1,5$.

As médias seguidas das mesmas letras, na coluna, não diferem entre si, pelo teste de Tukey, a $5 \%$ de probabilidade. 


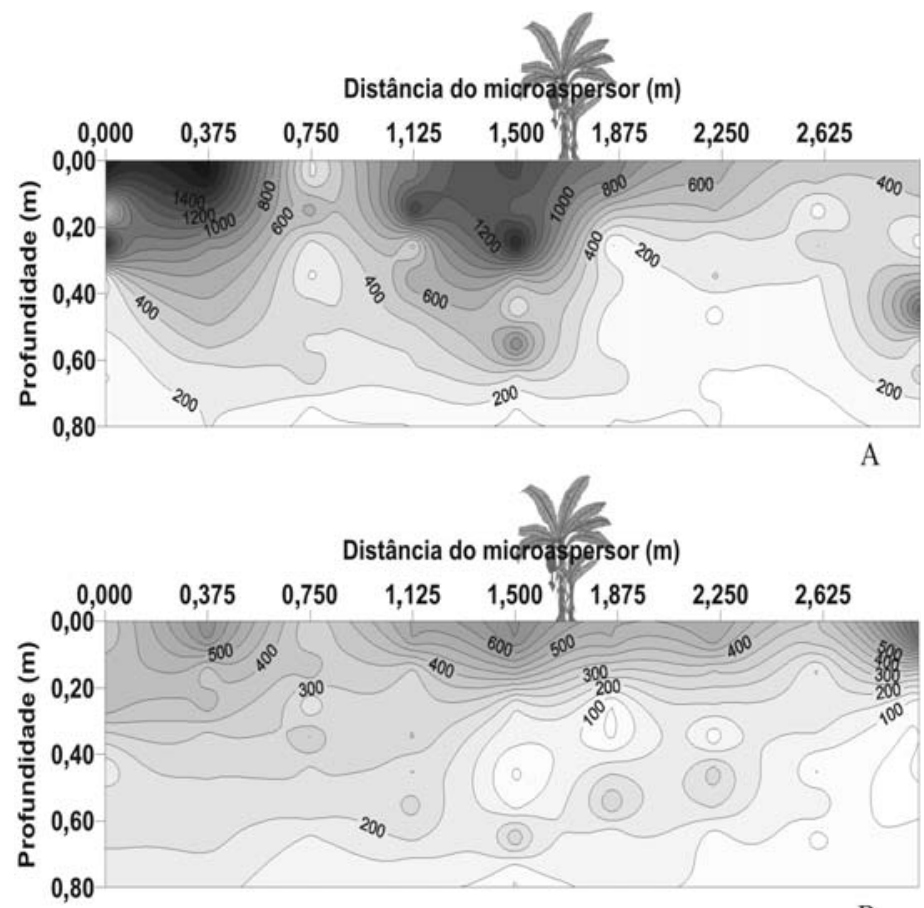

FIGURA 1 - Distribuição espacial da densidade total de raízes da bananeira, em cm de raiz/ $\mathrm{dm}^{\mathrm{B}} \mathrm{de}$ solo, em função da profundidade e da distância do microaspersor (ponto 0,000), nas freqüências de 3 dias (A) e 15 dias (B) de fertirrigação com uréia. Cruz das Almas-BA.

TABELA 2 - Densidade de raízes da bananeira 'Prata-Anã', por classes de diâmetro, em função da profundidade do solo. Média de duas freqüências de fertirrigação e de nove distâncias do microaspersor. Cruz das Almas-BA.

\begin{tabular}{|c|c|c|c|c|c|c|c|c|}
\hline \multirow{2}{*}{$\begin{array}{l}\text { Profundidade } \\
\text { (m) }\end{array}$} & \multicolumn{8}{|c|}{ Diâmetro das raízes $(\mathrm{mm})$} \\
\hline & D1 & D2 & D3 & D4 & D5 & D6 & D7 & TOTAL \\
\hline $0-0,10$ & 13,1 a & 27,3 a & $162,1 \mathrm{a}$ & 146,0 a & 128,9 a & $118,4 \mathrm{a}$ & $144,3 \mathrm{a}$ & 740,1 a \\
\hline $0,20-0,30$ & $7,7 \mathrm{~b}$ & $16,0 \mathrm{bc}$ & $103,2 \mathrm{abc}$ & $93,1 \mathrm{ab}$ & $70,1 \mathrm{bc}$ & $60,2 \mathrm{bc}$ & $69,3 \mathrm{~b}$ & $419,5 \mathrm{bc}$ \\
\hline $0,30-0,40$ & $6,9 \mathrm{~b}$ & $12,8 \mathrm{bc}$ & $73,5 \mathrm{bc}$ & $56,8 \mathrm{bc}$ & $46,1 \mathrm{~cd}$ & $48,4 \mathrm{bcd}$ & $47,8 \mathrm{bc}$ & $292,5 \mathrm{~cd}$ \\
\hline $0,40-0,50$ & $6,8 b$ & $13,7 \mathrm{bc}$ & $78,9 \mathrm{bc}$ & $58,1 \mathrm{bc}$ & $41,6 \mathrm{~d}$ & $36,7 \mathrm{~cd}$ & $33,6 \mathrm{~cd}$ & $269,3 \mathrm{~cd}$ \\
\hline $0,70-0,80$ & $3,2 \mathrm{c}$ & $5,9 \mathrm{~d}$ & $32,6 \mathrm{~d}$ & $22,3 \mathrm{~d}$ & $16,6 \mathrm{e}$ & $14,7 \mathrm{f}$ & $15,4 \mathrm{e}$ & $110,6 \mathrm{e}$ \\
\hline
\end{tabular}

${ }^{1}$ Classes de diâmetro $(\mathrm{mm}): \mathbf{D} 1=<0,1 ; \mathbf{D} 2=0,1-0,2 ; \mathbf{D 3}=0,2-0,3 ; \mathbf{D} 4=0,3-0,5 ; \mathbf{D 5}=0,5-0,8 ; \mathbf{D 6}=0,8-1,5$ e $\mathbf{D} 7=>1,5$.

As médias seguidas das mesmas letras, na coluna, não diferem entre si, pelo teste de Tukey, a $5 \%$ de probabilidade.

TABELA 3 - Densidade de raízes da bananeira 'Prata- Anã', por classes de diâmetro, em função da distância do microaspersor. Média de duas freqüências de fertirrigação e de oito profundidades. Cruz das Almas- BA.

\begin{tabular}{|c|c|c|c|c|c|c|c|c|}
\hline \multirow{2}{*}{$\begin{array}{l}\text { Distância } \\
\text { (m) }\end{array}$} & \multicolumn{8}{|c|}{ Diâmetro das raízes (mm) } \\
\hline & D1 & D2 & D3 & D4 & D5 & D6 & D7 & TOTAL \\
\hline & \multicolumn{8}{|c|}{-- Densidade de raízes $\left(\mathrm{cm}\right.$ de $\mathrm{raiz} / \mathrm{dm}^{3}$ de solo) -- } \\
\hline O & $9,4 \mathrm{ab}$ & $19,0 \mathrm{ab}$ & $110,5 \mathrm{a}$ & $105,7 \mathrm{ab}$ & $81,3 \mathrm{ab}$ & 58,9 abc & $29,0 \mathrm{e}$ & $413,8 \mathrm{abcd}$ \\
\hline 0,375 & $10,7 \mathrm{a}$ & $22,3 \mathrm{a}$ & $134,2 \mathrm{a}$ & $122,0 \mathrm{a}$ & $95,9 \mathrm{a}$ & $78,6 \mathrm{a}$ & 73,2 abc & $536,9 \mathrm{a}$ \\
\hline 0,750 & $6,7 \mathrm{abc}$ & $12,5 \mathrm{bcd}$ & 73,1 abc & $53,1 \mathrm{cde}$ & 44,0 cde & $45,0 \mathrm{bcd}$ & 43,0 cde & 277,5 cde \\
\hline 1,125 & $8,7 \mathrm{ab}$ & 15,4 abc & $92,5 \mathrm{ab}$ & $79,7 \mathrm{abcd}$ & $71,5 \mathrm{abc}$ & $71,6 \mathrm{ab}$ & $87,1 \mathrm{ab}$ & $426,4 \mathrm{abc}$ \\
\hline 1,500 & $9,6 \mathrm{a}$ & 17,3 abc & $109,4 \mathrm{a}$ & 92,2 abc & 72,8 abc & $71,9 \mathrm{ab}$ & 137,6 a & $510,8 \mathrm{ab}$ \\
\hline 1,850 & $5,3 \mathrm{bc}$ & $10,4 \mathrm{~cd}$ & $56,5 \mathrm{bc}$ & 46,0 def & 40,8 def & $40,8 \mathrm{~cd}$ & 55,9 abcd & $255,8 \mathrm{cde}$ \\
\hline 2,225 & $6,3 \mathrm{abc}$ & $13,2 \mathrm{abcd}$ & 76,3 abc & 43,2 ef & 31,7 ef & $35,6 \mathrm{~cd}$ & $32,6 \mathrm{de}$ & $239,0 \mathrm{de}$ \\
\hline 2,600 & $4,1 \mathrm{c}$ & $8,3 \mathrm{~d}$ & $45,1 \mathrm{c}$ & $29,3 \mathrm{f}$ & $25,5 \mathrm{f}$ & $28,7 \mathrm{~d}$ & $37,3 \mathrm{de}$ & $178,2 \mathrm{e}$ \\
\hline 2,975 & $6,8 \mathrm{abc}$ & $14,4 \mathrm{abcd}$ & $83,6 \mathrm{ab}$ & 64,2 bcde & 47,8 bcde & $41,7 \mathrm{bcd}$ & 44,6 bcde & 303,2 bcde \\
\hline
\end{tabular}

${ }^{1}$ Classes de diâmetro $(\mathrm{mm}): \mathbf{D} 1=<0,1 ; \mathbf{D 2}=0,1-0,2 ; \mathbf{D 3}=0,2-0,3 ; \mathbf{D} 4=0,3-0,5 ; \mathbf{D 5}=0,5-0,8 ; \mathbf{D 6}=0,8-1,5$ e $\mathbf{D} 7=>1,5$. A planta está localizada a $1,675 \mathrm{~m}$ do microaspersor (distância 0)

As médias seguidas das mesmas letras, na coluna, não diferem entre si, pelo teste de Tukey, a $5 \%$ de probabilidade. 


\section{REFERÊNCIAS}

AVILAN R., L.; MENESES R., L.; SUCRE, R.E. Distribución radical del banano bajo diferentes sistemas de manejo de suelos. Fruits, Paris, v.37, n.2, p.103-110,1982.

GARCIA, R. V. Sistema radicular de bananeira irrigada por aspersão convencional e microaspersão no Projeto Jaíba-MG.

2000. 47 f. Dissertação (Mestrado em Solos e Nutrição de Plantas)

- Universidade Federal de Viçosa, Viçosa, 2000.

GUDDANTI, S.; CHAMBERS, J. L. Gsroot automated root length measurement program, version 5.00: user's manual. Louisiana: Louisiana State University, 1993. 40 p.
MONTENEGRO, A.A.T.; GONDIM, R.S.; BEZERRA, M.A.; COSTA, C.A.G.; SABINO, K.V. Distribuição do sistema radicular da bananeira na microrregião do baixo Jaguaribe, Ceará. In: CONGRESSO BRASILEIRO DE FRUTICULTURA, 18., Florianópolis- SC. Anais... Florianópolis: SBF, 2004. 1CD-ROM.

SAS INSTITUTE. Statistical Analysis System Institute. SAS/ STAT user's guide: version $6.4^{\text {th }}$ ed. Cary, N C: 1990. v. 2, 1686 p.

SOUZA, L. da S.; SOUZA, L.D. Caracterização físico-hídrica de solos da área do Centro Nacional de Pesquisa de Mandioca e Fruticultura Tropical. Cruz das Almas: Embrapa Mandioca e Fruticultura, 2001. 56p. (Boletim de Pesquisa e Desenvolvimento, 20). 